\title{
Modelling of reaction cross sections and prompt neutron emission
}

\author{
F.-J. Hambsch ${ }^{1, a}$, A. Tudora ${ }^{2}$, and S. Oberstedt ${ }^{1}$ \\ 1 EC-JRC, Institute for Reference Materials and Measurements, Retieseweg 111, 2440 Geel, Belgium \\ 2 Bucharest University, Faculty of Physics, PO Box MG-11, 76900 Bucharest-Magurele, \\ Romania
}

\begin{abstract}
Accurate nuclear data concerning reaction cross sections and the emission of prompt fission neutrons (i.e. multiplicity and spectra) as well as other fission fragment data are of great importance for reactor physics design, especially for the new Generation IV nuclear energy systems. During the past years for several actinides $\left({ }^{238} U(n, f)\right.$ and ${ }^{237} \mathrm{~Np}(\mathrm{n}, \mathrm{f})$ ) both the reaction cross sections and prompt neutron multiplicities and spectra have been calculated within the frame of the EFNUDAT project.
\end{abstract}

\section{Introduction}

Accurate nuclear data are very important ingredients for reactor physics design, especially for the new Generation IV nuclear energy system projects. Not only are reaction cross sections of importance but also information on prompt fission neutron emission data (i.e. multiplicities and spectra). In addition information about the reaction products, i.e. fission fragment properties (in the case of neutron-induced fission) is also highly valuable and most of the time these data are input parameters in theoretical model calculations. Very important quantities are emission yields as a function of fragment mass and kinetic energy. The theoretical prediction of the fission mass yield distributions is a long-standing item, maybe as old as it is the understanding of the fission process itself. Up to now the theoretical description of the fission process is not able to give a reasonable prediction of the mass yield in comparison with the experimental data. The present theoretical mass distributions are too narrow and give as most probable heavy fragment mass a value around 132, while experimentally a most probable mass around 140 is found.

Based on the multi-modal random-neck rupture model [1] and fission cross section calculations we have tried to predict the fission fragment mass yield distributions for three different systems ${ }^{237} \mathrm{~Np}(\mathrm{n}, \mathrm{f})$, ${ }^{235} \mathrm{U}(\mathrm{n}, \mathrm{f})$ and ${ }^{238} \mathrm{U}(\mathrm{n}, \mathrm{f})[2]$. At that time the experimental data base on fission fragment mass and kinetic energy distributions was rather limited at low incident neutron energy. In the meantime new measurements have been performed [3], which unfortunately could not verify the predictions from Ref. [2].

Hence new and improved calculations have been performed for ${ }^{238} \mathrm{U}(\mathrm{n}, \mathrm{f})$ and the calculations have been extended for ${ }^{237} \mathrm{~Np}(\mathrm{n}, \mathrm{f})$ which are described in the following chapters.

\footnotetext{
${ }^{a}$ e-mail: franz-josef.hambsch@ec.europa.eu
}

This is an Open Access article distributed under the terms of the Creative Commons Attribution-Noncommercial License 3.0, which permits unrestricted use, distribution, and reproduction in any noncommercial medium, provided the original work is properly cited. 


\section{Modelling}

\subsection{Reaction cross sections}

2.1.1 ${ }^{238} \mathrm{U}(\mathrm{n}, \mathrm{f})$
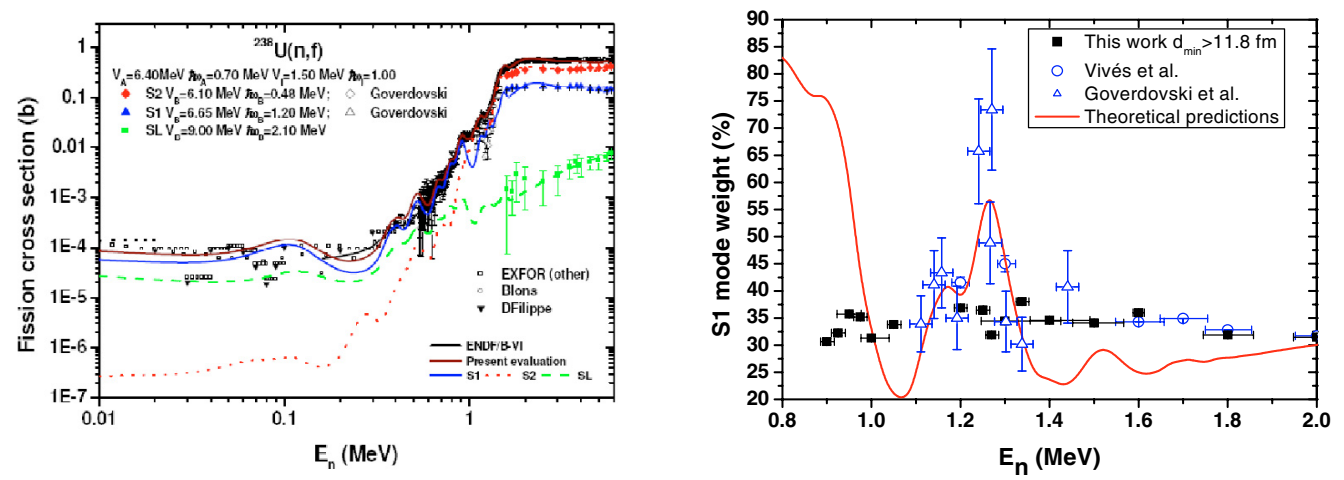

Fig. 1. Left part: The total fission cross section in comparison with mode separated fission cross sections for ${ }^{238} \mathrm{U}(\mathrm{n}, \mathrm{f})$ together with the corresponding calculated mode separated fission cross sections (broken lines). Right part: The predicted modal branching ratio for the Standard 1 mode (full line) as a function of incident neutron energy [1] compared to the most recent experimental data (full points) of Ref. [3].

Several reaction cross section calculations for different deformed actinide targets have been already performed (see e.g. Ref. [4] and references therein), so the applied model has a proven record of successful applications and comparison with experimental data. Here we emphasize only a few improvements brought to the different aspects of the modelling in the cases of ${ }^{238} \mathrm{U}$ and ${ }^{237} \mathrm{~Np}$ neutron induced reactions.

The improved calculation of the ${ }^{238} \mathrm{U}$ neutron induced reaction cross-section takes into account the most recent coupled-channel deformed optical model parameterizations and recently reported values for the experimental s-wave neutron average level spacing and mean gamma width as well as a refined model for fission. The fission barrier parameter values used are in agreement with all theoretical and statistical considerations. The obtained integral and differential cross-sections give a very good description of all existing experimental data.

Concerning the direct interaction (DI) mechanism treated by the coupled channel (CC) method, in Ref. [2] we have used the deformed potential of Vladuca et al. [5]. This time we explored three new CC parameterisations (Soukhovitsky et al. [6], BRC [7] and Capote et al. [8]), to choose the deformed potential giving the best consistent agreement with existing experimental data for the total cross-section, differential elastic and inelastic cross-sections at incident energies were the compound nucleus mechanism contribution becomes negligible. The best description of the experimental data was obtained for the following two cases: Vladuca parameterization [5] and Capote parameterization [8] with deformation parameters of Möller and Nix [9].

The compound nucleus $(\mathrm{CN})$ mechanism is treated within the statistical model with sub-barrier effects using the STATIS code, in the version including the extended fission model in the frame of the multi-modal fission concept [10] in case of ${ }^{238} \mathrm{U}$. This extended theory of the statistical model including the competition with three fission modes is described in detail in previous papers (see for instance Ref. [11]). In the fission mode concept the same inner barrier and isomeric well for all modes is considered, only the outer barriers being different for each mode, because theoretical calculations showed that the bifurcation point is placed at deformation shapes de-passing the second well [12]. The left part of Figure 1 shows the previous result of the fission cross-section calculation in terms of fission modes for ${ }^{238} U(n, f)[2]$. Based on this result the modal weights of the so-called standard fission modes 

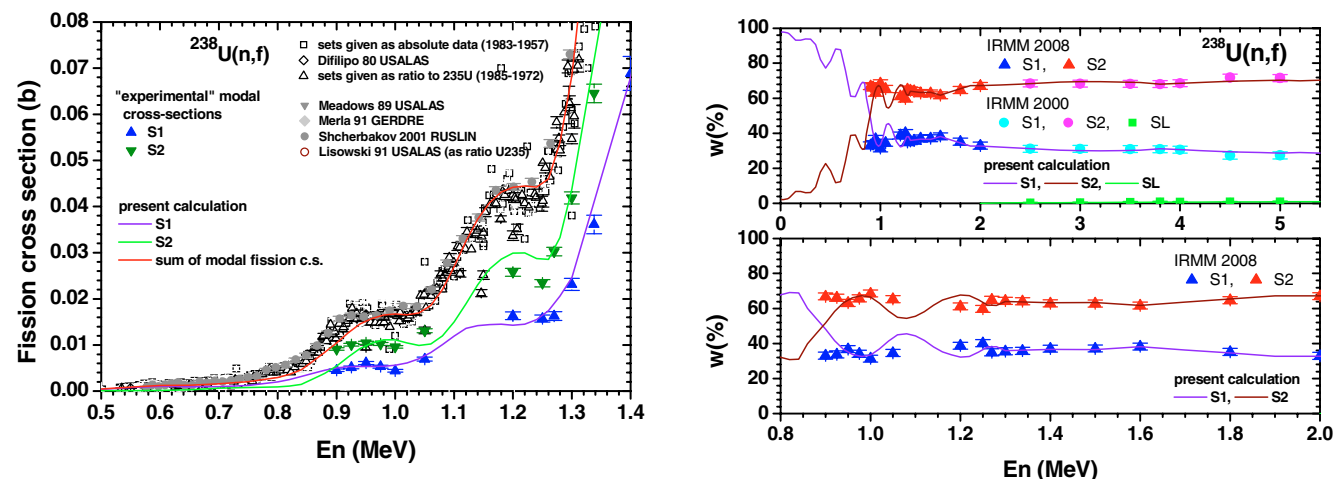

Fig. 2. Left part: New fission cross section calculation for the S1 and S2 modes as well as their sum (full lines) in comparison with the experimental data in the vicinity of the vibrational sub-barrier resonances.
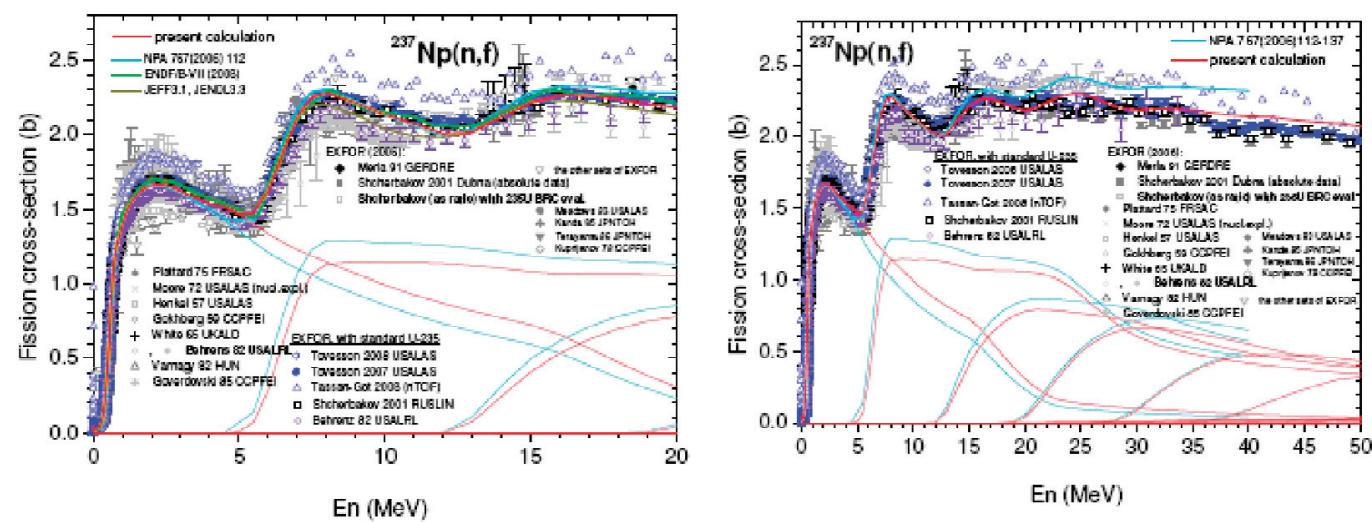

Fig. 3. The fission cross section for ${ }^{237} \mathrm{~Np}(\mathrm{n}$, f), left part in the incident neutron energy range up to $20 \mathrm{MeV}$, right part up to $50 \mathrm{MeV}$. The coloured full lines give the contribution of the different chances and are compared to older calculations.

can be calculated. The previous modal weight predictions given in the right part of Figure 1 as the full red line are based on experimental data mainly concentrated around the vibrational resonance at about $1.25 \mathrm{MeV}$ incident neutron energy [2]. The new experimental results of Ref. [3] however do not show the predicted strong fluctuation of the standard 1 fission mode. Figure 2 finally shows the improved calculations mentioned above. The left part of Figure 2 shows the contributions of the main fission modes (standard 1 and 2) to the fission cross section. The right part of Figure 2 gives the fission mode weights for standard 1 (S1) and standard 2 (S2) as a function of incident neutron energy, the upper part in the full studied incident energy range and the lower part as a zoom into the neutron energy range from 0.8 to $2 \mathrm{MeV}$. So by changing slightly the barrier parameter values in the model calculations also the most recent experimental results could be reproduced.

\subsection{2 ${ }^{237} \mathrm{~Np}(\mathrm{n}, \mathrm{f})$}

The model calculations of ${ }^{237} \mathrm{~Np}$ neutron-induced cross-sections, prompt fission neutron multiplicity and spectra as well as of other quantities related to the prompt neutron emission had already been done up to an incident neutron energy of $40 \mathrm{MeV}$ (see Ref. [13]). In the present work the calculations (without fission modes) have been extended up to an incident neutron energy of $50 \mathrm{MeV}$ also including the fission of the secondary uranium nucleus chain formed by charged particle emission. The resulting fission cross-section is plotted in Figure 3 in comparison with experimental data taken from EXFOR. The left part shows the incident neutron energy region up to the standard value of $20 \mathrm{MeV}$ used in 


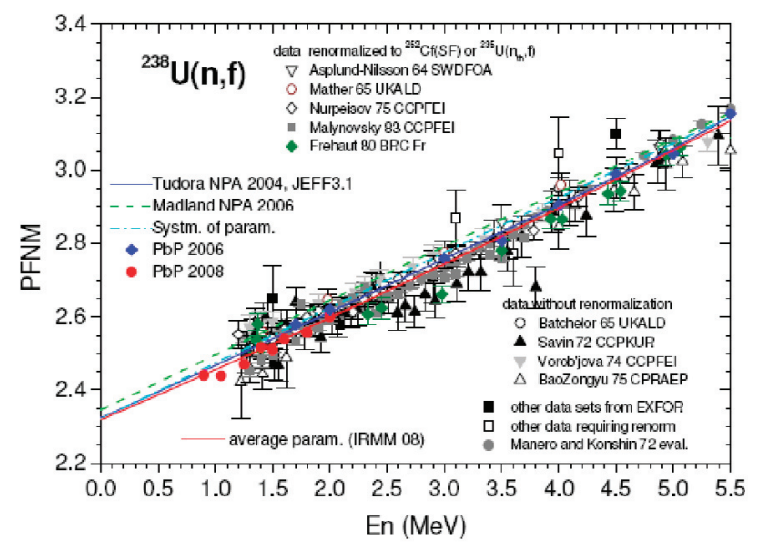

Fig. 4. The PbP multiplicity calculations (red full points) compared to previous results and experimental data.

evaluated nuclear data files. In the right part the fission cross-section result in the entire studied energy range (up to $50 \mathrm{MeV}$ ) is plotted. The present calculation is compared to our previous one (full blue and red lines). In addition the contributions of the different chances are also given both from the previous calculation (Ref. [13], blue lines) and the present calculation (red lines).

\subsection{Prompt neutron emission}

\subsection{1 ${ }^{238} U(n, f)$ and ${ }^{237} \mathrm{~Np}(\mathrm{n}, \mathrm{f})$}

The prompt neutron emission is treated in the frame of the Point by Point $(\mathrm{PbP})$ model that takes into account the full range of possible fragmentations of the fissioning nucleus (details are given in Refs. [14-16]). The PbP approach provides all prompt fission quantities related to the fission fragment (FF) and fragment pairs, the so-called multi-parametric matrix $v(\mathrm{~A}, \mathrm{TKE})$. In this manner the total average prompt neutron multiplicity (PFNM) and spectrum (PFNS), as well as other average quantities related to the prompt fission process, are calculated as superposition of the respective quantities of the FF pairs weighted with the FF distributions.

The PbP model is used in the case of spontaneous fission (SF) and neutron induced fission in the incident energy range where only one compound nucleus $(\mathrm{CN})$ undergoing fission is formed, because of the difficulty to take into account many fragmentations of the secondary fissioning nuclei (formed at higher incident energy) having a continuous range of excitation energies. Moreover, usually for the FF mass and total kinetic energy distributions, that are input quantities in the PbP model, experimental data are preferred. Above the first chance fission threshold it is impossible to infer from the experimental data the particular $\mathrm{CN}$ undergoing fission. In addition, at high incident neutron energies the experimental Y(A, TKE) data are scarce or totally absent. In the energy range where multiple fission chances are involved the "most probable fragmentation" approach is used with average model parameter values obtained from the PbP treatment (details are given in Refs. [17,18] and references therein).

The present multiplicity results obtained by the PbP model calculation and the equivalent most probable fragmentation approach (using average input model parameters based on the PbP treatment) are plotted in Figure 4 for ${ }^{238} \mathrm{U}(\mathrm{n}, \mathrm{f})$ and in the left part of Figure 5 for ${ }^{237} \mathrm{~Np}(\mathrm{n}, \mathrm{f})$ in comparison with experimental data from EXFOR. Previous results $[15,18]$ are also given for comparison.

The present ${ }^{237} \mathrm{~Np}$ multiplicity result in the full neutron energy range up to $50 \mathrm{MeV}$ obtained by using the extended prompt neutron emission model (described in Ref. [17]) that takes into account the fission of secondary nucleus chains formed by charged particle emission is plotted in the right part of Figure 5 in comparison with experimental data taken from EXFOR. The contributions of the main and secondary compound nucleus chains and path are also given in the figure. 

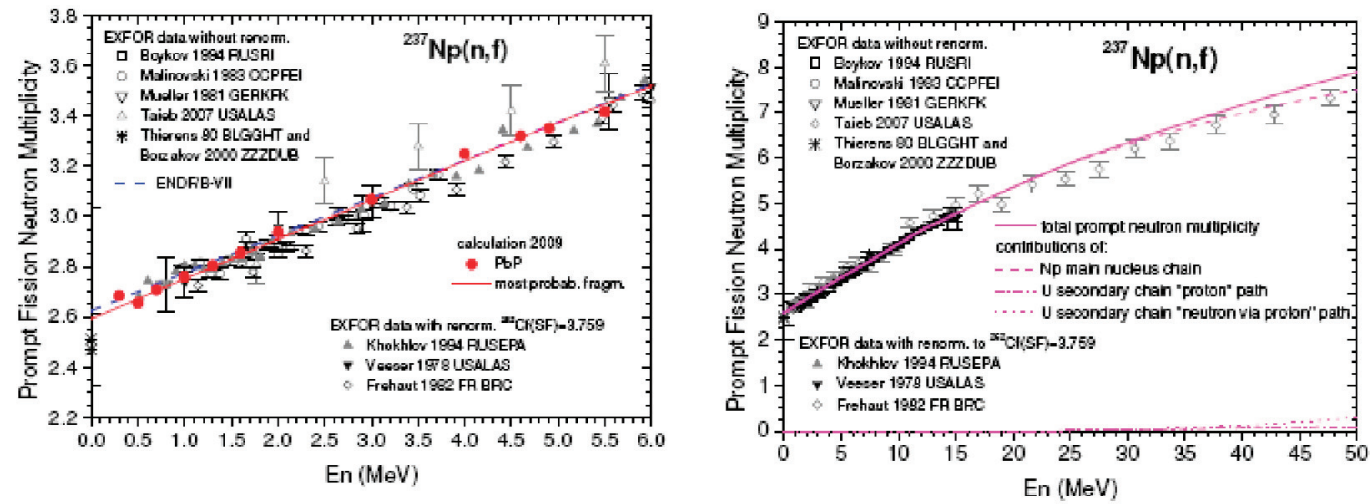

Fig. 5. Prompt neutron multiplicity calculation for ${ }^{237} \mathrm{~Np}(\mathrm{n}, \mathrm{f})$, left part up to 6 , right part up to $50 \mathrm{MeV}$ incident neutron energy and compared to experimental data.

\section{Conclusions}

Improved calculations both for the reaction cross sections including fission as well as prompt neutron multiplicity have been done for ${ }^{238} \mathrm{U}(\mathrm{n}, \mathrm{f})$ and ${ }^{237} \mathrm{~Np}(\mathrm{n}, \mathrm{f})$. For both nuclides an improvement of the reaction cross sections has been achieved, which is in better agreement with the experimental data with better justified barrier parameters and a better coupled channel parameterisation.

For the prompt neutron emission multiplicities and spectra the point by point model has been used for both nuclides also improving the quality of the agreement between experimental data and new calculations. This is due to the fact that also the secondary nucleus chain contribution and improved fission chance probabilities have been taken into account in case of ${ }^{237} \mathrm{~Np}(\mathrm{n}, \mathrm{f})$.

One of us (AT) would like to acknowledge the support of the EFNUDAT project (contract number 036434).

\section{References}

1. U. Brosa, S. Grossmann, A. Müller, Phys. Rep. 197167 (1990)

2. F.-J. Hambsch, G. Vladuca, A. Tudora, S. Oberstedt, I. Ruskov, Ann. Nucl. Energy 321297 (2005)

3. E. Birgersson, A. Oberstedt, S. Oberstedt, F.-J. Hambsch, Nucl. Phys. A817 1 (2009)

4. E. Rich, A. Tudora, G. Noguère, J. Tommasi, J.-F. Lebrat, Nucl. Sci. Eng. 162171 (2009) and G. Vladuca, F.-J. Hambsch, A. Tudora, S. Oberstedt, A. Oberstedt, F. Tovesson, D. Filipescu Nucl. Phys. A740 3 (2004)

5. G. Vladuca, A. Tudora, M. Sin, Rom. J. Phys. 41515 (1996) and IAEA Reference Input Parameter Library RIPL-1, Segment IV, iref=600

6. E. Soukhovitsky, S. Chiba, J.-Y. Lee, O. Iwamoto, T. Kukahori, Phys. Rev. G30 905 (2004)

7. B. Morillon, P. Romain, Phys. Rev. C70 014601 (2004)

8. R. Capote, E. Soukhovitskii, J.M. Quesada, S. Chiba, Phys. Rev. C72 064610 (2005)

9. RIPL-2 and RIPL-1 libraries, Möller and Nix data base

10. G. Vladuca, A. Tudora, F.-J. Hambsch, S. Oberstedt, I. Ruskov, Nucl. Phys. A720 274 (2003)

11. G. Vladuca, A. Tudora, F.-J. Hambsch, S. Oberstedt, Nucl. Phys. A707 32 (2002)

12. S. Oberstedt, F.-J. Hambsch, F. Vivés, Nucl. Phys. A644 289 (1998)

13. G. Vladuca, Anabella Tudora, B. Morillon, D. Filipescu, Nucl. Phys. A767 112 (2006)

14. Anabella Tudora, Ann. Nucl. Energy 351 (2008)

15. Anabella Tudora, Ann. Nucl. Energy 331030 (2006)

16. Anabella Tudora, F.-J. Hambsch, Ann. Nucl. Energy 37771 (2010)

17. A. Tudora, G. Vladuca, B. Morillon, Nucl. Phys. A740 33 (2004)

18. Anabella Tudora, Ann. Nucl. Energy 3672 (2009) 